\title{
Predicting spike times of a detailed conductance-based neuron model driven by stochastic spike arrival
}

\author{
Renaud Jolivet *, Wulfram Gerstner \\ School of Computer and Communication Sciences and Brain-Mind Institute, \\ Ecole Polytechnique Fédérale de Lausanne (EPFL), 1015 Lausanne, Switzerland
}

\begin{abstract}
Reduced models of neuronal activity such as integrate-and-fire models allow a description of neuronal dynamics in simple, intuitive terms and are easy to simulate numerically. We present a method to fit an integrate-and-fire-type model of neuronal activity, namely a modified version of the spike response model, to a detailed Hodgkin-Huxley-type neuron model driven by stochastic spike arrival. In the Hogkin-Huxley model, spike arrival at the synapse is modeled by a change of synaptic conductance. For such conductance spike input, more than $70 \%$ of the postsynaptic action potentials can be predicted with the correct timing by the integrate-and-fire-type model. The modified spike response model is based upon a linearized theory of conductance-driven integrate-and-fire neurons.
\end{abstract}

(c) 2005 Elsevier Ltd. All rights reserved.

PACS: 87.10.+e; 87.19.La; 87.17.Nn; 87.17.Aa

Keywords: Conductance injection; Integrate-and-fire model; Stochastic input; Mapping technique; Predictive power

\section{Introduction}

The seminal work by Hodgkin and Huxley [13], on the mathematical description of action potential generation, has led to a whole series of models that describe in detail the dynamics of various ionic currents and the effect of the dendritic architecture on signal integration, see e.g. $[1,6,23,33]$. However, the precise description of neuronal activity involves a large number of variables, which often prevents a clear understanding of the underlying dynamics. Hence, a simplified description is desirable and has been subject of numerous studies (for a review, see [12,21]). The most popular simplified models include the integrateand-fire model [19,32], the FitzHugh-Nagumo model $[9,25]$ and the Morris-Lecar model [24]. However, it is not clear if such simplified models are sufficient to capture

\footnotetext{
* Corresponding author.

E-mail address: renaud.jolivet@epfl.ch (R. Jolivet).

$U R L$ : http://icwww.epfl.ch/ rjolivet (R. Jolivet).
}

the essence of neuronal dynamics. Indeed, reduced models of neuronal activity, as opposed to detailed models of the Hodgkin-Huxley-type, are commonly thought to be too simple to account for the rich firing behavior of real neurons.

Nevertheless, these highly simplified models have been shown to yield good predictions when compared to biological data $[16,28]$. In particular, several parameter estimation and/or optimization techniques have been proposed to map reduced models to real neurons [14-16,27,28]. These techniques allow to map electrophysiological data from biological neurons (intracellular or extracellular recordings) onto simplified models of the integrate-andfire-type. Moreover, such a mapping could be the starting point of a systematic classification of cortical neurons in terms of simplified dynamics. Finally, reduced models have been used extensively and successfully to model and study analytically the behavior of cortical-like networks $[4,11]$. In other words, analytical and computational tools are available to go from a simple description of single cells dynamics to network dynamics. 
In this paper, we review and extend a numerical technique that allows a systematic mapping of a class of integrate-and-fire neuron models, namely the spike response model $[12,17]$, to intracellular recordings of neuronal activity [15]. While this technique ensures reliable predictions and good generalization when the target neuron is driven by a randomly fluctuating current, it was shown in a previous study [15] that the generalization power is poor when the target neuron is driven by randomly fluctuating synaptic conductances. Hence, the purpose of this paper is to propose extensions of our previous work which address the latter problem. Using recent theoretical results [29,31], we propose a generalization of the spike response model so as to make model parameters input dependent. This improved version of the SRM is shown to be very efficient and robust at predicting the spike train of a detailed Hodgkin-Huxley-type neuron model.

\section{Materials and methods}

\subsection{The spike response model}

We consider a neuron stimulated by stochastic presynaptic spike arrival. The state of the neuron is characterized by a single variable $u$, the membrane voltage of the cell at the soma. Let us suppose that the neuron has fired its last spike at time $\hat{t}$. At time $t>\hat{t}$, the membrane potential of the cell is described by

$u(t)=\eta(t-\hat{t})+\sum_{i \in E} \sum_{f} \epsilon^{+}\left(t-t_{i}^{f}\right)+\sum_{j \in I} \sum_{f} \epsilon^{-}\left(t-t_{j}^{f}\right)$

The last two terms account for the drive by presynaptic neurons $i$ (respectively $j$ ) of the excitatory $E$ (respectively inhibitory $I$ ) population. $t_{i}^{f}$ and $t_{j}^{f}$ denote the firing time of presynaptic neurons. The $\epsilon$-functions describe the excitatory $(+)$ and inhibitory $(-)$ postsynaptic potentials (EPSPs and IPSPs). $\eta(t-\hat{t})$ describes the form of the spike itself as well as the afterhyperpolarization potential, if present. A spike is elicited if the following threshold condition is satisfied

if $u(t) \geqslant \vartheta(t-\hat{t})$ and $\quad \frac{\mathrm{d} u}{\mathrm{~d} t}>0$ then, $\hat{t}=t$

Note that spiking occurs only if the membrane voltage crosses the threshold $\vartheta$ from below. The threshold itself can be taken either as a constant or as time dependent. In this paper, we use a dynamic threshold of the form

$$
\vartheta(t-\hat{t})= \begin{cases}+\infty & \text { if } t-\hat{t} \leqslant \gamma_{\text {ref }} \\ \vartheta_{0}+\vartheta_{1} \exp \left[-(t-\hat{t}) / \tau_{\vartheta}\right] & \text { else }\end{cases}
$$

where $\gamma_{\text {ref }}$ is a fixed absolute refractory period so as to exclude continuous firing. $\vartheta_{0}, \vartheta_{1}$ and $\tau_{\vartheta}$ are parameters that will be chosen to yield the best fit to a target spike train (see Section 2.2). This version of the spike response model (the one that we use in this paper) is a simplified version of the full spike response model and has been termed $\mathrm{SRM}_{0}$ (see [12] for further details). We will use this acronym to refer to this model.

Eq. (1) can be restated in the form

$u(t)=\eta(t-\hat{t})+\int_{0}^{+\infty} \epsilon^{+}(s) Q^{+}(t-s) \mathrm{d} s+\int_{0}^{+\infty} \epsilon^{-}(s) Q^{-}(t-s) \mathrm{d} s$

with

$Q^{+}(t)=\sum_{i \in E} \sum_{f} \delta\left(t-t_{i}^{f}\right), \quad Q^{-}(t)=\sum_{j \in I} \sum_{f} \delta\left(t-t_{j}^{f}\right)$
For numerical implementation, we will use a discrete version of $Q$ 's

$Q_{t}^{+,-}=\int_{t}^{t+\Delta t} Q^{+,-}(s) \mathrm{d} s$

i.e. $Q_{t}^{+}$is the spike count in a time bin of duration $\Delta t$ in the excitatory presynaptic population and analogously for $Q_{t}^{-}$. The activity (population rate) $A^{+}$of the excitatory presynaptic population is defined as follows:

$A^{+}(t)=\frac{1}{\Delta t} \frac{\int_{t}^{t+\Delta t} Q^{+}(s) \mathrm{d} s}{N^{+}}$

where $N^{+}$is the size of the presynaptic population (with a corresponding definition for the inhibitory population). All simulations are done using a fixed time bin $\Delta t=0.2 \mathrm{~ms}$.

\subsection{Mapping the $S R M_{0}$ to voltage traces}

The mapping technique that we propose has been discussed in detail elsewhere [15] and we refer interested readers to this reference. Here, we describe the essentials of the technique without going into details.

To realize the mapping of the $\mathrm{SRM}_{0}$ to the target neuron, we proceed in two steps. First, we extract the functions characterizing the model (EPSP $\epsilon^{+}$, IPSP $\epsilon^{-}$and spike shape $\eta$ ) and second, we choose the parameters of the dynamic threshold $(\vartheta)$ and optimize them in terms of quality of predictions. To do so, we assume that we have at our disposal voltage traces of the target neuron as well as firing times of presynaptic neurons. We also assume that the input characteristics are kept constant during the recording of the dataset used for the mapping procedure. We start by extraction of the spike shape $\eta$. The shape of spikes is usually highly stereotyped and presents only little variability. We therefore select one spike train from the dataset and align all spikes relatively to some arbitrarily chosen initiation point. The mean trajectory of the spikes yields $\eta$. Detection and alignment of spikes is realized using a threshold condition on the first derivative of the membrane voltage. Once we are done with $\eta$, we extract the shape $\epsilon^{+}$of an EPSP and the shape $\epsilon^{-}$of an IPSP. If we limit ourselves to the interval between two consecutive spikes of the same spike train $\hat{t}_{k}$ and $\hat{t}_{k+1}$, we can rewrite Eq. (1) with the notation introduced in Eq. (4) as follows (for $\hat{t}_{k}<t<\hat{t}_{k+1}$ )

$u(t)-\eta\left(t-\hat{t}_{k}\right)=\int_{0}^{+\infty} \epsilon^{+}(s) Q^{+}(t-s) \mathrm{d} s+\int_{0}^{+\infty} \epsilon^{-}(s) Q^{-}(t-s) \mathrm{d} s$

It is then possible to find the optimal $\epsilon$-functions using the Wiener-Hopf optimal filtering technique [20,34]. Resulting $\epsilon$-functions are optimal in the sense that they minimize the mean square distance between the predicted membrane voltage $u(t)$ and the membrane voltage of the target neuron given the same input. We fit the resulting EPSP $\epsilon^{+}$with a suitable function, typically a difference of exponentials

$\epsilon^{+}(s)=K_{\epsilon}^{+}\left[\exp \left(-s / \tau_{r}^{+}\right)-\exp \left(-s / \tau_{d}^{+}\right)\right]$

with a corresponding definition for $\epsilon^{-} . K_{\epsilon}, \tau_{r}$ and $\tau_{d}$ are free parameters. The final step of model optimization is to choose and optimize the threshold. The absolute refractory period $\gamma_{\text {ref }}$ is set to $2 \mathrm{~ms}$. The other parameters of Eq. (3), i.e. $\vartheta_{0}, \vartheta_{1}$ and $\tau_{\vartheta}$, are fitted in order to optimize the coincidence factor $\Gamma$ (see below). In order to ensure a good generalization, we optimize the threshold with a large dataset generated with different input characteristics set apart for parameter optimization. The numerical optimization algorithm that we use is the downhill simplex method [26]. Obviously, the $\mathrm{SRM}_{0}$ can only predict neuronal activity of the specific neuron it has been mapped to.

\subsection{Target neuron model}

Instead of real data from experiments, we use as a reference or "target" a Hodgkin-Huxley-type model of a fast-spiking interneuron [8]. It contains standard $\mathrm{Na}^{+}$and $\mathrm{K}^{+}$spike-generating channels and one extra $\mathrm{Kv} 1.3 \mathrm{~K}^{+}$-channel derived from "n"-type currents measured in human T-lymphocytes. The Kv1.3 current produces a subtle form of adaptation. See [8] for further details. 


\subsection{Input scenario}

The Hodgkin-Huxley-type model of a fast-spiking interneuron is driven by random synaptic conductances generated by massive stochastic presynaptic spike arrival. The total driving current is given by [30]

$I_{\text {syn }}(t)=g_{\text {syn }}^{+}(t)\left[u(t)-E^{+}\right]+g_{\text {syn }}^{-}(t)\left[u(t)-E^{-}\right]$

where $g_{\text {syn }}^{+,-}$are the excitatory $(+)$and inhibitory (-) conductances and $E^{+,-}$are the corresponding reversal potential. Synaptic conductances are generated by slightly correlated homogeneous Poisson spike trains. The excitatory and inhibitory populations contain respectively $N^{+}=8000$ and $N^{-}=2000$ neurons. These two populations are independent of each other. Spike trains of the $N^{+}$excitatory neurons are constructed from $\bar{N}^{+}<N^{+}$independent Poisson spike trains via duplication of spike times (see Appendix A for further details), analogously for inhibitory input. This procedure makes all the presynaptic neurons within a given population (i.e. excitatory or inhibitory) correlated with zero time lag. For a given target value of the correlation coefficient, $\bar{N}^{+,-}$depend on the presynaptic discharge frequency and on the size of the presynaptic population. In the following, we assume that the presynaptic neurons discharge at frequencies $v^{+}$and $v^{-}$and the correlation coefficient is held at a constant value $c=0.002$ (see Appendix A for further details about how the presynaptic spike trains are generated and, in particular, see Eqs. (A.11) and (A.12) for the relation between $\bar{N}^{+,-}$and the correlation coefficient $c$ ). This kind of correlated input ensures that the membrane voltage of the HodgkinHuxley-type model neuron exhibits large fluctuations. We have previously reported that the amplitude of fluctuations is a critical factor for the ability of threshold models to predict spikes with a correct timing based upon a strict threshold condition [15]. This may seem to be a strong constraint on our approach. However, the correlation coefficient chosen here is very small $(c=0.002)$. Moreover, reliable spike timing of cortical neurons in vitro is only possible if the input has sufficient fluctuations [22].

The dynamics of each excitatory synapse $i$ is described by a conductance variable $P_{i}^{+}$with

$\tau_{\mathrm{syn}}^{+} \frac{\mathrm{d}}{\mathrm{d} t} P_{i}^{+}=-P_{i}^{+}+D^{+} \sum_{f} \delta\left(t-t_{i}^{f}\right)$

The value of $P_{i}^{+}$is increased by an amount $D^{+}$for each presynaptic spike activating the synapse at time $t_{i}^{f}$. It then decays back to zero with a time constant $\tau_{\text {syn. }}^{+}$. The total excitatory conductance is the sum of conductance variables $P_{i}^{+}$over all excitatory synapses. Thus, the total excitatory conductance is

$g_{\text {syn }}^{+}(t)=\sum_{i \in E} P_{i}^{+}(t)=\frac{D^{+}}{\tau_{\text {syn }}^{+}} \int_{0}^{+\infty} \mathrm{e}^{-s / \tau_{\text {syn }}^{+}} Q^{+}(t-s) \mathrm{d} s$

with a corresponding definition for the total inhibitory conductance. Numerical values used in this paper are summarized in Table 1.

\subsection{Assessing the quality of predictions of the reduced model}

In order to evaluate quantitatively the predictions of our reduced model, we use the coincidence factor $\Gamma$ between two spike trains [17] defined as

$\Gamma=\frac{N_{\text {coinc }}-\left\langle N_{\text {coinc }}\right\rangle}{\frac{1}{2}\left(N_{\text {target }}+N_{\mathrm{SRM}}\right)} \frac{1}{\mathscr{N}}$

Table 1

Parameters of excitatory and inhibitory synapses (adapted from Destexhe and Paré [6])

\begin{tabular}{lcll}
\hline Synapse & $E(\mathrm{mV})$ & $\tau_{\text {syn }}(\mathrm{ms})$ & $D\left(\mathrm{mS} / \mathrm{cm}^{2}\right)$ \\
\hline Excitatory $(+)$ & 0 & 2.45 & 0.073 \\
Inhibitory $(-)$ & -80 & 6.11 & 0.04 \\
\hline
\end{tabular}

Parameter $D$ has been adjusted so as to yield an amplitude of postsynaptic potential of the order of $1 \mathrm{mV}$. where $N_{\text {target }}$ is the number of spikes in the target spike train $S_{\text {target }}, N_{\mathrm{SRM}}$ is the number of spikes in the spike train $S_{\mathrm{SRM}}$ that is predicted by our reduced model, $N_{\text {coinc }}$ is the number of coincidences with precision $\Delta$ between the two spike trains, and $\left\langle N_{\text {coinc }}\right\rangle=2 v \Delta N_{\text {target }}$ is the expected number of coincidences generated by a homogeneous Poisson process with the same rate $v$ as the spike train $S_{\mathrm{SRM}}$. The factor $\mathscr{N}=1-2 v \Delta$ normalizes $\Gamma$ to a maximum value of one which is reached if and only if the spike train of the reduced model reproduces exactly that of the target neuron. A homogeneous Poisson process with the same frequency as the reduced model would yield, on average, $\Gamma=0$. We compute the coincidence factor $\Gamma$ by comparing the two complete spike trains, i.e., the spike train $S_{\text {target }}$ generated by the target neuron and the train $S_{\mathrm{SRM}}$ predicted by the SRM. This is different to the approach of Kistler and colleagues [17] where $\Gamma$ was used to predict the next spike in a spike train, under the assumption that past action potentials were correctly reconstructed. Note that the coincidence factor $\Gamma$ is similar to the "reliability" as defined in [22]. In the present paper, the precision $\Delta$ was fixed to a constant value $\Delta=2 \mathrm{~ms}$ that is supposed to be approximately the width of a spike. Note that $\Delta$ is different from $\Delta t$, the time bin used for simulations.

\subsection{Linearized theory of a conductance-driven integrate-and-fire model}

The results of Section 3.1 show that a SRM $\mathrm{SM}_{0}$ with fixed time course of EPSPs $\epsilon^{+}$and IPSPs $\epsilon^{-}$has a rather limited range of validity. The naive solution would therefore be to use a different set of EPSPs and IPSPs for each set of discharge frequencies $\left\{v^{+} ; v^{-}\right\}$. However, this is not a very practical solution if we have to compute PSPs $\epsilon^{+}$and $\epsilon^{-}$for each possible combination of input frequencies with the method indicated above. Instead, we aim at finding a simple parameterization of the EPSPs and IPSPs that would allow to interpolate between and generalize beyond the specific inputs used for the numerical derivation of $\epsilon^{+}$and $\epsilon^{-}$. To do so, we use a linearized theory of conductance-driven integrate-and-fire models that allows to write down an analytical expression for the EPSPs $\epsilon^{+}$and IPSPs $\epsilon^{-}$in function of the discharge frequencies $v^{+}$and $v^{-}$. Thus, the extended spike response model $\mathrm{SRM}_{\mathrm{c}}$ that we derive in this paper is directly related to the subthreshold dynamics of a conductance-driven integrate-and-fire neuron model (CIF) [5,29,31].

The subthreshold membrane voltage $u$ of a CIF neuron model is given by the following differential equation:

$C \frac{\mathrm{d}}{\mathrm{d} t} u=-g_{\mathrm{L}}\left(u-E_{\mathrm{L}}\right)-g_{\text {syn }}^{+}(t)\left[u-E^{+}\right]-g_{\text {syn }}^{-}(t)\left[u-E^{-}\right]$

with $C$ the membrane capacitance, $g_{\mathrm{L}}$ the leak conductance (with a reversal potential $E_{\mathrm{L}}$ ) and $g_{\text {syn }}^{+}$(respectively $g_{\text {syn }}^{-}$) the instantaneous excitatory (respectively inhibitory) conductance. $E^{+}$and $E^{-}$are the reversal potentials of the excitatory and inhibitory synapses. It is straightforward to show that the average membrane voltage predicted by this model (in absence of spiking mechanism) is given by

$\mu_{\mathrm{CIF}}=\frac{g_{\mathrm{L}} E_{\mathrm{L}}+\bar{g}_{\text {syn }}^{+} E^{+}+\bar{g}_{\text {syn }}^{-} E^{-}}{g_{\mathrm{L}}+\bar{g}_{\text {syn }}^{+}+\bar{g}_{\text {syn }}^{-}}$

The bars denote time averaging in this case. For example, if $N^{+}$presynaptic neurons fire at rate $v^{+}$, we have

$\bar{g}_{\text {syn }}^{+}=D^{+} N^{+} v^{+} \tau_{\text {syn }}^{+}$

The EPSPs $\epsilon^{+}$and IPSPs $\epsilon^{-}$of the $\mathrm{SRM}_{0}$ that would correspond to the CIF model can then be calculated as the linear response around the average membrane voltage $\mu_{\mathrm{CIF}}$. We find

$\epsilon^{+}(s)=\frac{D^{+} \tau_{\text {eff }} \tau_{\text {syn }}^{+}\left(\mu_{\text {CIF }}-E^{+}\right)}{\tau_{\text {eff }}-\tau_{\text {syn }}^{+}}\left(\mathrm{e}^{-s / \tau_{\text {syn }}^{+}}-\mathrm{e}^{-s / \tau_{\text {eff }}}\right)$

with a corresponding definition for $\epsilon^{-}(s)$. $\tau_{\text {eff }}$ is the effective membrane time constant and is given by $\tau_{\text {eff }}=C /\left(g_{\mathrm{L}}+\bar{g}_{\text {syn }}^{+}+\bar{g}_{\text {syn }}^{-}\right)$. Note that Eq. (17) is equivalent to a current-based approximation. We compare the results of Eqs. (15) and (17) with results extracted from simulations of the target Hodgkin-Huxley-type neuron model. We find that, outside the 
regime of EPSP-amplification, both the average membrane voltage (data not shown) and the shape of PSPs $\epsilon^{+}$and $\epsilon^{-}$are well predicted by this simple linearized theory (see Fig. 2B in Section 3). To illustrate this, we fit the PSPs plotted in Fig. 1A with Eq. (17) with $\tau_{\text {eff }}$ as a free parameter. We then compare this parameter with $\tau_{\text {eff }}$ as predicted by the Eq. (19). Fig. 3 shows that the linearized theory is in very good agreement with the results of simulations of the target Hodgkin-Huxley-type neuron model except in the area where inhibition is weak.

Eq. (17) gives an analytical expression for the PSPs $\epsilon^{+}$and $\epsilon^{-}$. The PSPs allow us to reproduce the fluctuations of the membrane voltage. However, we also need to account for the constant voltage bias which appears when discharge frequencies are elevated. Therefore, we redefine the first term of the $\mathrm{SRM}_{0}$, namely the function $\eta$ as

$\eta(t-\hat{t}) \rightarrow \eta(t-\hat{t})+\left(\mu_{\mathrm{CIF}}-E_{\mathrm{L}}\right)$
This simple procedure ensures that our model produces the correct average membrane voltage. The model could be further improved by using a time-dependent leak conductance $g_{\mathrm{L}}(t-\hat{t})$ [15], but we will not do so.

In order to relate the linearized theory of conductance-driven integrate-and-fire model of Section 3.2 to the numerical PSPs $\epsilon^{+}$and $\epsilon^{-}$ extracted in Section 3.1, we need to estimate the parameters appearing in Eq. (17) from the data. We will assume that the size of the presynaptic populations $\left(N^{+}\right.$and $\left.N^{-}\right)$as well as the average discharge frequencies in these populations $\left(v^{+}\right.$and $\left.v^{-}\right)$are known. We will also assume "standard" reversal potentials at the synapses, i.e. $E^{+}=0 \mathrm{mV}$ and $E^{-}=-80 \mathrm{mV}$. $C$ is taken to be constant at a value of $1 \mu \mathrm{F} / \mathrm{cm}^{2}$. The reversal potential of the leak current can be crudely approximated by the resting potential of the neuron $E_{\mathrm{L}} \approx u_{\text {rest }}$.

Thus, the parameters we need to estimate are the synaptic time constants $\tau_{\text {syn }}^{+}$and $\tau_{\text {syn }}^{-}$, the effective membrane time constant $\tau_{\text {eff, }}$, as well as

A
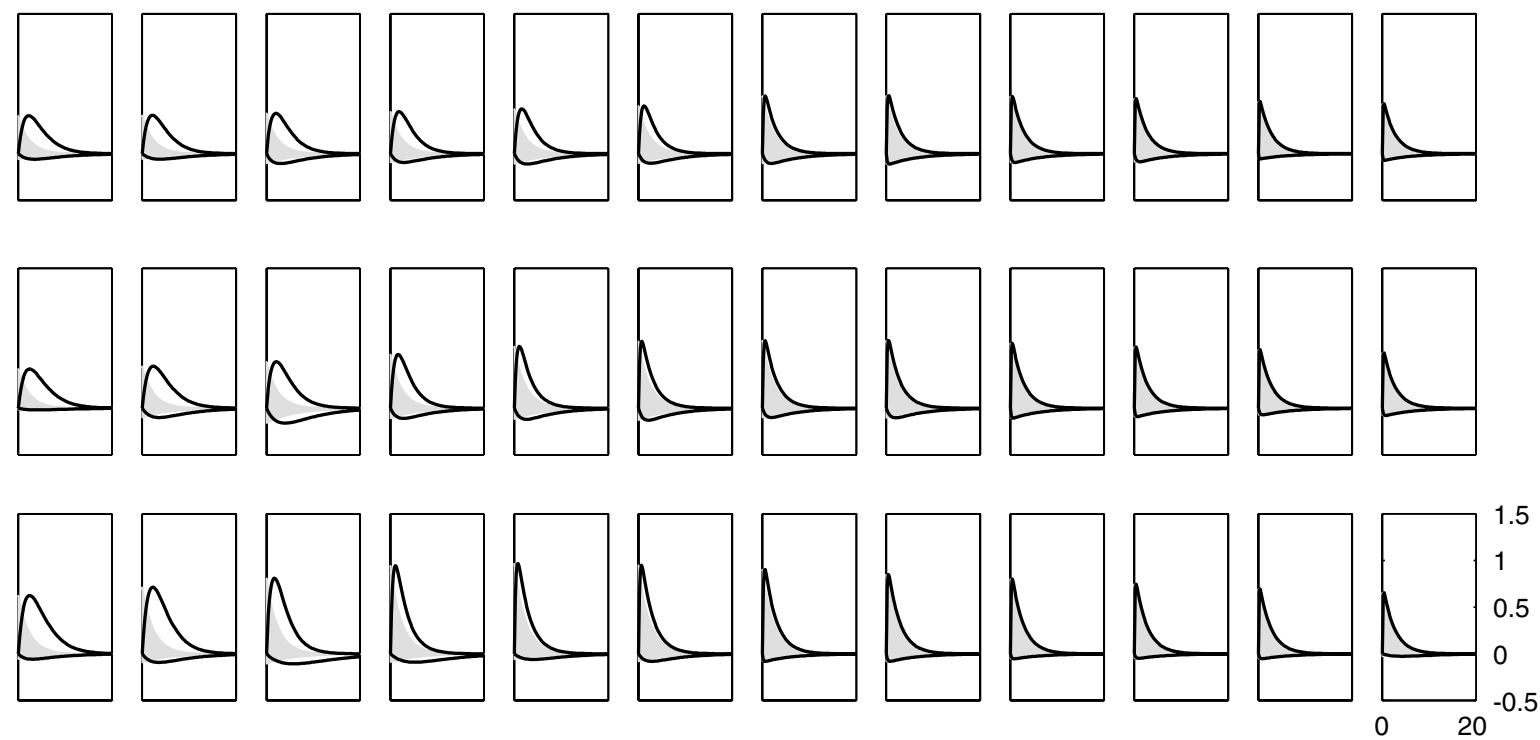

B

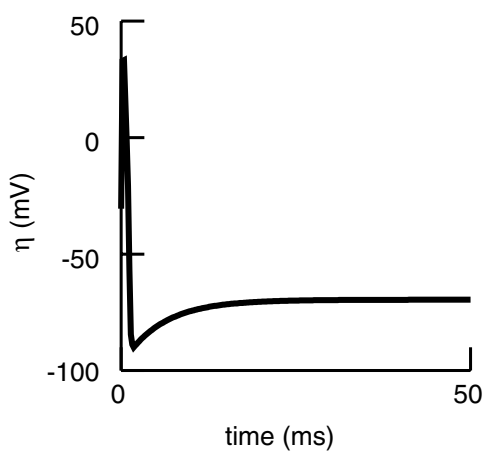

C

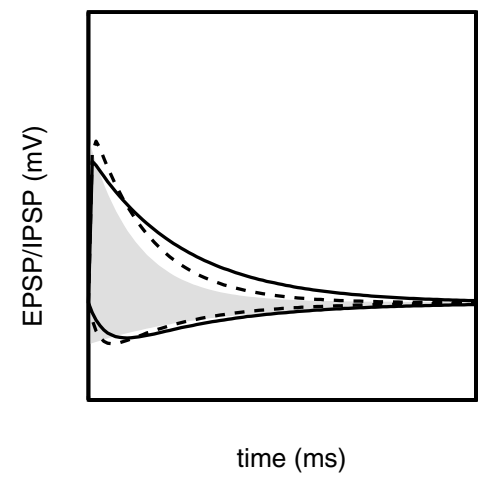

D 
the mean conductances $g_{\mathrm{L}}, \bar{g}_{\text {syn }}^{+}$and $\bar{g}_{\text {syn }}^{-}$. The time constants $\tau_{\text {syn }}^{+}$(respectively $\tau_{\text {syn }}^{-}$) can be estimated by fitting the EPSP $\epsilon^{+}$(respectively the IPSP $\epsilon^{-}$) extracted from a dataset where EPSP-amplification does not take place, i.e. a dataset with strong inhibition and weak excitation. Once $\tau_{\text {syn }}^{+}$and $\tau_{\text {syn }}^{-}$are known, one can find the other parameters by comparing EPSPs $\epsilon^{+}$extracted for several different sets of input characteristics. Fitting the EPSPs $\epsilon^{+}$with formula (17) yields an estimate of $\tau_{\text {eff }}$. If we collect three different values of $\tau_{\text {eff }}$ for three different sets of input characteristics $\left(v^{+}\right.$and $\left.v^{-}\right)$and using the definition of $\tau_{\text {eff }}$ introduced above, we find a set of three equations with three unknowns, namely $g_{\mathrm{L}}, \bar{g}_{\text {syn }}^{+}$and $\bar{g}_{\text {syn }}^{-}$where the average $\bar{g}_{\text {syn }}^{+}$is given in Eq. (16). Solving this set of equations thus yields an estimate of $g_{\mathrm{L}}, D^{+}$and $D^{-}$. Therefore, we now have a simple model that allows to compute PSPs $\epsilon^{+}(s)$ and $\epsilon^{-}(s)$ in a straightforward fashion for any given set of input discharge frequencies $v^{+}$and $v^{-}$. We will refer to this model as $\mathrm{SRM}_{\mathrm{c}}$ with a lower-case "c" for conductance-based. The advantage of using this two-step procedure, i.e. first a non-parametric extraction of the PSPs and then a fit by parametric functions, is the following. The non-parametric extraction provides a straightforward and robust estimate of the PSPs. Then, the resulting PSPs are fitted with a simple parametric function and comparison of the results obtained with three different sets of input discharge frequencies provides a direct estimate of the model parameters. The overall procedure is much simpler than a direct non-linear parametric optimization technique.

\section{Results}

In the first subsection, we discuss the intrinsic limitations of the simple spike response model $\mathrm{SRM}_{0}$. In particular, we show that the shape of EPSPs and IPSPs derived by our method changes systematically as a function of the input parameters. These findings are then compared with a linearized theory of conductance-driven integrateand-fire models (CIF). This comparison allows us to determine the parameters of a new conductance-based spike response model $\left(\mathrm{SRM}_{\mathrm{c}}\right)$ which is tested over a broad range of different inputs in Section 3.2.

\subsection{Limitations of a classic $S R M_{0}$}

We map the target neuron model to the $\mathrm{SRM}_{0}$ using the technique described in Section 2. Let us recall that the $\mathrm{SRM}_{0}$ is characterized mainly by the spike shape $\eta$ as well as the EPSP $\epsilon^{+}$and the IPSP $\epsilon^{-}$. Fig. 1A shows the EPSPs and IPSPs $\epsilon^{+,-}$extracted for different input discharge frequencies $v^{+}$and $v^{-}$and Fig. 1B shows the spike shape $\eta$. The shape of the spike does not depend on the characteristics of the input scenario. One immediately remarks that both the characteristic time scales and the amplitude of the EPSP/IPSP do change in function of the input discharge frequencies. In fact, the numerical technique extracts the best linear filters so as to reproduce the membrane voltage trace of a given sample spike train. The corresponding EPSPs and IPSPs are then optimal for the specific set of input discharge frequencies used for parameter extraction but there is no reason why they should be optimal for other sets of inputs with different characteristics. Indeed, there are reasons why they should be different depending on input discharge frequencies. Let us quickly review these reasons [6].
First, two different sets of input discharge frequencies are likely to produce two different values of average membrane voltage. In turn, this means increased or decreased average driving forces for the synapses as the corresponding current includes a multiplicative term of the form $u(t)-E^{+,-}$(see Eq. (10)). This should affect the amplitude of the EPSPs and IPSPs. Second, two different sets of input discharge frequencies are likely to produce two different total conductances. This affects the effective membrane time constant of the neuron. If we neglect the effect of somatic AP-generating ion channels, the effective time constant in the subthreshold regime can be written

$$
\tau_{\text {eff }}=\frac{C}{g_{\mathrm{L}}+\bar{g}_{\text {syn }}^{+}+\bar{g}_{\text {syn }}^{-}}
$$

with $C$ the membrane capacitance, $g_{\mathrm{L}}$ the conductance of the leak current and $\bar{g}_{\text {syn }}^{+}$and $\bar{g}_{\text {syn }}^{-}$the total average excitatory and inhibitory synaptic conductances. Finally, when the total excitatory drive is large, the target neuron runs in a highly non-linear regime due to activation of $\mathrm{Na}^{+}$-channels. This effect is known as EPSP-amplification [10] and illustrated for our target neuron in Fig. 1C and D. While EPSP-amplification is usually not observed in fast-spiking interneurons, it is present in our target model of a fast-spiking interneuron since $\mathrm{Na}^{+}$-channels are the only channels opened at depolarized states close to threshold (see Section 2). All these three effects (average driving force, effective membrane time constant and EPSP-amplification) combine with each other and lead to the pattern of EPSPs and IPSPs shown in Fig. 1A and C. The EPSPs and IPSPs are shorter when total conductance is increased (shortening of effective membrane time constant) except when this increase is mainly due to the excitatory conductance which then leads to the reverse effect because of EPSP-amplification (see panel C). The amplitude of EPSPs decreases when excitation is increased and increases when excitation is decreased (average driving force) with corresponding effects for IPSPs and modifications of the inhibition level. Interestingly, one remarks that when inhibition is very strong together with weak excitation, the extracted EPSPs $\epsilon^{+}$and IPSPs $\epsilon^{-}$follow exactly the dynamics of the corresponding synapses, i.e. an almost instantaneous rise followed by a decay with the same time constant as the time constant of the synaptic conductance (see Fig. 1A at bottom right). The dynamics of EPSPs and IPSPs can be approximated by

$\epsilon^{+,-}(s) \propto\left(\mathrm{e}^{-s / \tau_{\text {syn }}^{+,-}}-\mathrm{e}^{-s / \tau_{\text {eff }}}\right)$

Therefore, when drive is very strong, $\tau_{\text {eff }} \approx 0$ from Eq. (19) and $\epsilon^{+,-}$is proportional to $\mathrm{e}^{-s / \tau_{\text {syn }}^{+-}}$. Note that this effect does appear only with weak excitatory stimulation. When excitatory drive is strong, EPSP-amplification compensates the reduction of $\tau_{\text {eff }}$ and Eq. (19) does not hold anymore.

Given the change of the time course of EPSPs and IPSPs as a function of the input, it is clear that correct predictions of the subthreshold fluctuations of the membrane voltage by linear summation of EPSPs and IPSPs with a fixed time 
course are only possible in the input regime for which the reduced model was constructed (recall Eq. (1)).

In order to make the EPSPs and IPSPs input dependent, we parameterize $\epsilon^{+}$and $\epsilon^{-}$using the linearized theory of a conductance-driven integrate-and-fire model [5,29,31]; see Section 2 for details.

We find that outside the regime of EPSP-amplification the numerically derived postsynaptic potentials $\epsilon^{+}$and $\epsilon^{-}$ are well fitted by the theory (see Fig. 2). The regime of EPSP-amplification can easily be identified by comparing the effective membrane time constant $\tau_{\text {eff }}$ predicted by the theory with that derived from the numerically optimized PSPs $\epsilon^{+}$and $\epsilon^{-}$(see Fig. 3). All parameters of the linearized theory, in particular the synaptic time constants $\tau_{\text {syn }}^{+}, \tau_{\text {syn }}^{-}$ and the mean conductances $\bar{g}_{\text {syn }}^{+}$and $\bar{g}_{\text {syn }}^{-}$can hence be estimated from the data by using three sets of inputs that do not lead to EPSP-amplification (see Section 2). Note that although extraction of the PSPs $\epsilon^{+}$and $\epsilon^{-}$requires stationary input statistics, the resulting conductance-driven spike response model $\mathrm{SRM}_{\mathrm{c}}$ allows to take into account changes in the input frequency. In a network simulation where spike times are not known in advance, the instantaneous input frequencies $v^{+}$and $v^{-}$can be approximated by the instantaneous population activities in the presynaptic
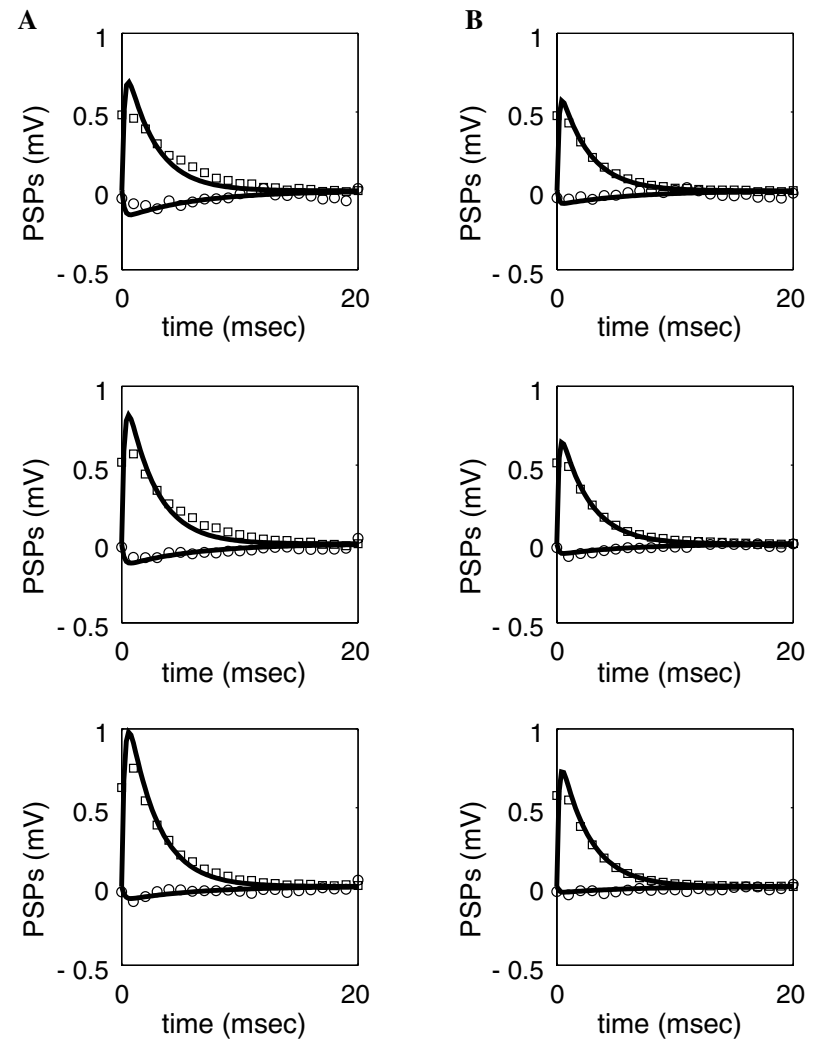

Fig. 2. The EPSPs and IPSPs as predicted by the linearized theory (solid lines; see Eq. (17)) are compared to the EPSPs and IPSPs extracted by the method proposed in Section 2 (symbols). (A) The inhibitory discharge frequency $v^{-}=6 \mathrm{~Hz}$ and the excitatory discharge frequency $v^{+}=$ $0.9,0.6,0.3$ (from top to bottom). (B) Same as in (A) except that $v^{-}=$ $10 \mathrm{~Hz}$.

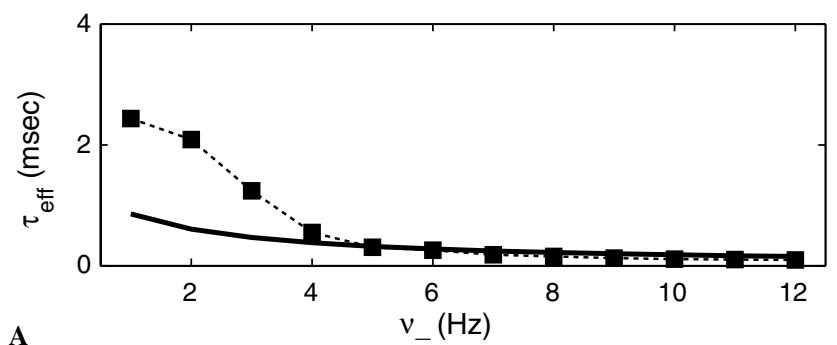

A
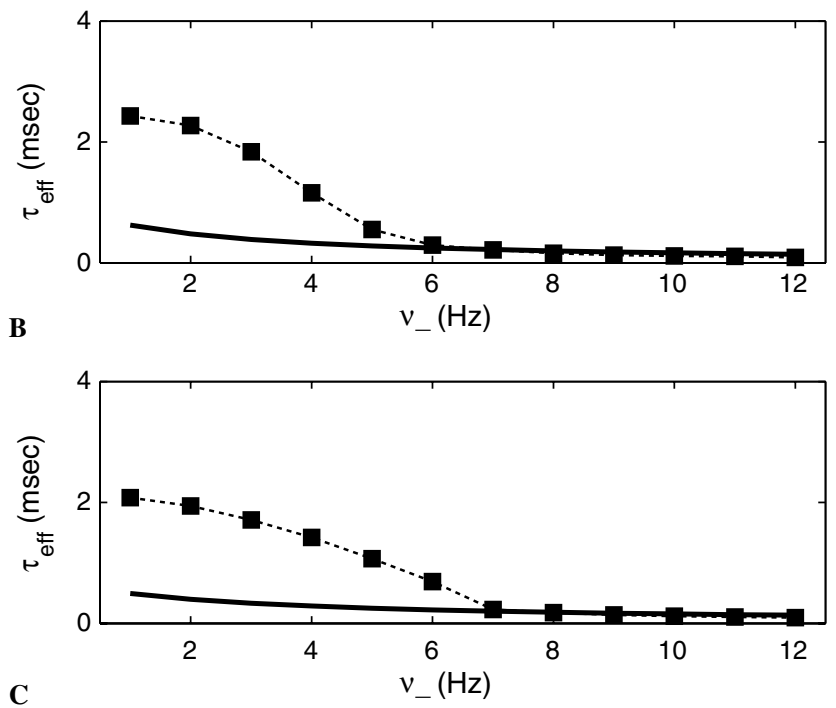

Fig. 3. The effective membrane time constant as predicted by a CIF (solid line) is compared to the corresponding parameter extracted from simulations of the target Hodgkin-Huxley-type neuron model (dotted line with squares). See text for further details. (A) $v^{+}=0.3 \mathrm{~Hz}$. (B) $v^{+}=0.6 \mathrm{~Hz}$. (C) $v^{+}=0.9 \mathrm{~Hz}$.

pools. The conductance-driven spike response model $\left(\mathrm{SRM}_{\mathrm{c}}\right)$ will now be tested on a new set of inputs independent from the one used for parameter optimization.

\subsection{Predicting spike by spike}

A conductance-driven spike response model $\left(\mathrm{SRM}_{\mathrm{c}}\right)$ has been estimated from the numerical voltage traces using the procedure described in Section 2. We now test the predictive power of the $\mathrm{SRM}_{\mathrm{c}}$. We are interested in reproducing the exact timing of the spikes of the target neuron. As we have not dealt yet with the threshold, the first step is to optimize the three free parameters of the threshold, namely $\vartheta_{0}, \vartheta_{1}$ and $\tau_{\vartheta}$ (see Eq. (3), $\gamma_{\text {ref }}$ is set at a constant value of $2 \mathrm{~ms}$ ). To do so, we use a very long spike train with input characteristics that include the discharge frequencies where EPSP-amplification does not take place (see Fig. 3). We then proceed as indicated in Section 2. Note that the data used to optimize the parameters of the threshold are not part of the test set, i.e. the dataset used to assess the predictive power of the reduced model. The error (i.e. $1-\Gamma$, see below) is usually slightly smaller on the dataset used for parameter optimization than on the test set. However, the difference is not significant indicating that there is no 
Table 2

Best fit parameters for the threshold (see text for further details)

\begin{tabular}{lll}
\hline Parameter & Mean & SD \\
\hline$\vartheta_{0}(\mathrm{mV})$ & -38.437 & 0.002 \\
$\vartheta_{1}(\mathrm{mV})$ & 564.0 & 0.7 \\
$\tau_{\vartheta}(\mathrm{ms})$ & 0.91 & 0.03 \\
\hline
\end{tabular}

Mean and standard deviation (SD) are computed from four optimizations with different initial conditions. The small standard deviation shows that the four optimization runs all converge to the same minimum.

systematic overfitting. The resulting numerical values are summarized in Table 2.

Fig. 4 shows sample results of the predictive power of the $\mathrm{SRM}_{\mathrm{c}}$ in two distinct regimes, low-drive (low presynaptic discharge frequencies, 1) and high-drive (high presynaptic discharge frequencies, 2). In both cases the predicted membrane voltage is reasonably close to the membrane voltage of the target neuron. Note also that all the spikes in plotted samples are reproduced with the exact timing (panel B). For these two cases, we find $\Gamma=0.76$ (low-drive) and $\Gamma=0.67$ (high-drive). The coincidence factor $\Gamma$ takes a value of 1 if $100 \%$ of spikes coincide and is normalized to 0 if coincidences are random (see Section 2).

To test the performances in a more systematic way, we quantify the predictions of the $\mathrm{SRM}_{\mathrm{c}}$ in terms of the timing of the spikes (coincidence factor $\Gamma$ ) and in terms of output frequencies of the model $\left(v_{\text {out }}\right)$ over a broad range of input characteristics $\left(v^{+}\right.$and $\left.v^{-}\right)$. Fig. 5 shows the performances of the $\mathrm{SRM}_{\mathrm{c}}$ for such a systematic procedure. We observe that the $\mathrm{SRM}_{\mathrm{c}}$ yields good performances $(\Gamma \geqslant 0.7$ and output frequency $v_{\text {out }}$ predicted in the correct range) over a broad range of input discharge frequencies. The only notable exception is when inhibitory discharge frequency is very high. In this regime, the output frequency $v_{\text {out }}$ is close to zero so that the number of spikes in a spike train is low. Our coincidence factor $\Gamma$ is not well suited for this case. However, we note that even if $\Gamma$ is low, the output frequency is predicted in the correct range. Furthermore, the subthreshold fluctuations of the membrane voltage are well reproduced (not shown).

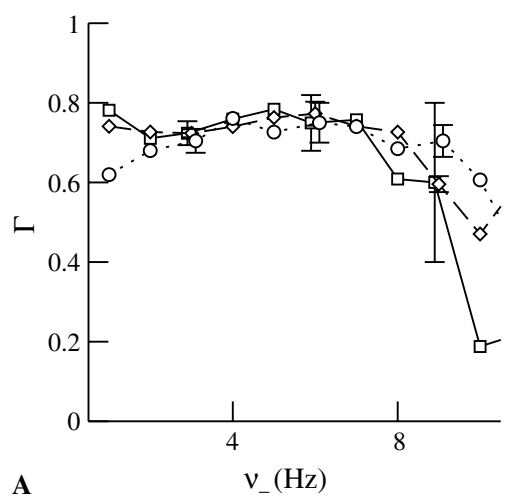

A. 1

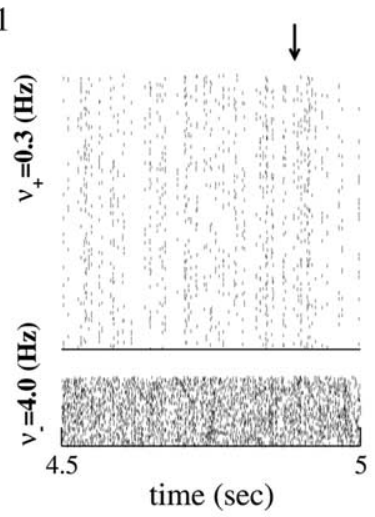

B.1

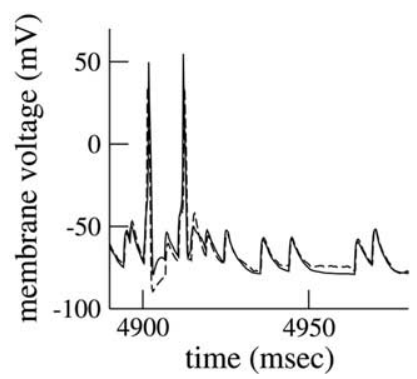

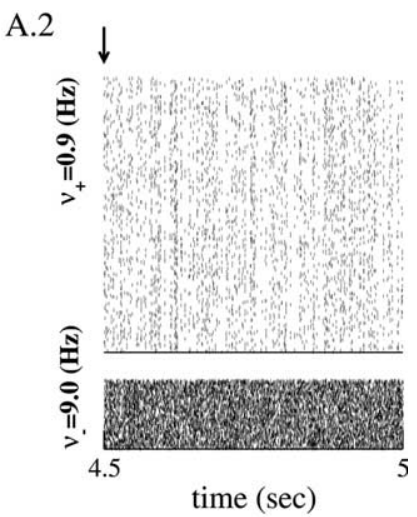

B.2

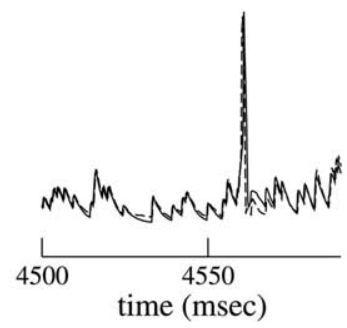

Fig. 4. Comparative results for low (1) and high (2) input discharge frequencies. (A) The activity in the excitatory (top) and inhibitory (bottom) presynaptic populations. The arrows indicate the starting point of the segment plotted in (B). (B) Corresponding membrane voltage of the target neuron (solid line) is compared to the membrane voltage as predicted by the $\mathrm{SRM}_{\mathrm{c}}$ (dashed line). In both cases (B.1 and B.2) the membrane voltage as predicted by the $\mathrm{SRM}_{\mathrm{c}}$ gives a fair approximation of the membrane voltage of the target neuron.

Interestingly, the $\mathrm{SRM}_{\mathrm{c}}$ yields good performances where it is supposed to do so but also where EPSP-amplification takes place (see Fig. 3). In the case of our target neuron, EPSP-amplification occurs when both excitatory and inhibitory populations discharge at rather low frequencies. A fixed coefficient of correlation $c$ then imposes co-activation of large subpopulations of synapses in this regime (see Eq. (A.11)). The target neuron therefore spends most of the

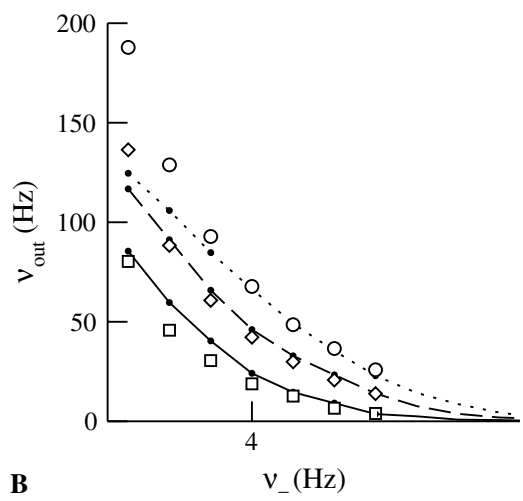

Fig. 5. Coincidence factor $\Gamma(\mathrm{A}$; mean $\pm \mathrm{SD})$ and output frequency $v_{\text {out }}(\mathrm{B})$ plotted versus the inhibitory input discharge frequency $v^{-}$for various values of the excitatory input discharge frequency $v^{+}\left(v^{+}=0.3 \mathrm{~Hz}\right.$ solid line and squares; $v^{+}=0.6 \mathrm{~Hz}$ dashed line and diamonds; $v^{+}=0.9 \mathrm{~Hz}$ dotted line and circles). In the panel $\mathrm{B}$, the output frequency of the $\mathrm{SRM}_{\mathrm{c}}$ (symbols) is compared to the output frequency of the target neuron (dots and line). 
time close to the resting potential and occasionally receives numerous coincident presynaptic spikes that bring it in the area of EPSP-amplification. Most of these large excursions then lead to a spike while only a few of them do not. In this situation, one can easily predict spikes at a correct timing with a threshold model like the $\mathrm{SRM}_{\mathrm{c}}$.

In summary, our modified version of the $\mathrm{SRM}_{0}$, the socalled $\mathrm{SRM}_{\mathrm{c}}$, performs well over a broad range of input characteristics $v^{+}$and $v^{-}$and predicts not only the output frequency but also most of the spikes with the correct timing. It is useful to keep in mind that we used a calculation based on a conductance-driven integrate-and-fire model to evaluate the subthreshold membrane voltage fluctuations and the average membrane voltage. The latter results do not include the potential effect of ionic channels activated in the subthreshold regime. The $\mathrm{SRM}_{\mathrm{c}}$ should therefore fail in regimes where such ionic channels are activated. However, we found here that even though the underlying theoretical framework is on the edge of its validity domain, the $\mathrm{SRM}_{\mathrm{c}}$ still performs reasonably well in the regime where EPSP-amplification takes place and thus as long as there are large enough fluctuations of the subthreshold membrane voltage.

\section{Discussion}

Mapping real neurons to simplified neuronal models has benefited from many theoretical developments in recent years and has been applied to both in vitro and in vivo recordings $[16,28]$. However, most of the techniques have been developed for a current injection scenario [3,2, $27,28]$. On the experimental side, conductance injection is increasingly used instead of current injection and is thought to be closer to in vivo conditions (see [7] for a review).

We had previously reported a mapping technique [15] based on standard signal processing tools which allows a systematic mapping of a simplified neuron model, the spike response model $[12,17]$, to intracellular recordings. It has been shown to yield very good results in the case of current injection for model neurons [15] and with in vitro data (unpublished observations). However, while the reduced model built in this way generalizes its predictions over a broad range of different input characteristics for the current injection case, it performs very poorly in the conductance injection case since, both the amplitude of the PSPs characterizing the model and their decay time constants depend on the total input conductance. A model using a single set of constant PSPs cannot account for such an input-dependent variability. Moreover, activation of $\mathrm{Na}^{+}$-channels produces a non-linear effect known as EPSP-amplification that affects the shape of PSPs. A linear model is likely to perform badly in such a highly non-linear regime.

In this paper, we have shown that a simple modification of the standard threshold model $\mathrm{SRM}_{0}$ solves this problem. In its new formulation, the model is able to predict very reliably many aspects of neuronal activity, such as timing of the spikes, membrane voltage and mean output rate. The new model $\mathrm{SRM}_{\mathrm{c}}$ is directly related to conductancedriven integrate-and-fire neurons [5,29,31]. Even though the two models are not strictly equivalent, it is a priori likely that a conductance-driven integrate-and-fire model would perform well too. Our technique is based upon direct estimation of effective EPSPs and IPSPs and can be applied to extract simple neuron models from experimental intracellular recordings under conductance injection.

\section{Acknowledgments}

The authors would like to thank Magnus Richardson for fruitful discussions. RJ is supported by the Swiss National Science Foundation under Grant No. N FN2100-065268.

\section{Appendix A. Generation of presynaptic spike trains}

In this appendix, we detail the method used in simulations to generate slightly correlated spike trains and we also derive some useful analytical results. The method follows [6] but there exists other ways to generate correlated spike trains (see [18] for instance).

Presynaptic spike trains are described by random homogeneous Poisson processes. At each time step, $\bar{N}$ independent random variables are generated and distributed among the $N \geqslant \bar{N}$ presynaptic neurons to generate slightly correlated spike trains [6]. In this specific case, we can derive the probability distribution function (PDF) of the variable $Q$ (see Eq. (6) in Section 2 for a definition of variable $Q$ ). Here, all the calculations rely on a discrete time scale with bins of width $\Delta t$. Let us consider that elements of a vector $\bar{V}$ of length $\bar{N}$ are distributed at random and receive either a value of 1 with a constant probability $p$ or 0 with probability $(1-p)$. In this specific case, $p=$ $v \Delta t$ with $v$ the average discharge frequency in the presynaptic population and $\Delta t$ the size of the time steps used in the simulation. In order for this procedure to generate Poisson spike trains, the probability $p$ must be small, i.e. $p \ll 1$. Therefore, $\Delta t$ must be chosen so that it is much smaller than the period $1 / v$ of the spike trains. The total number $K$ of elements receiving a value of 1 in $\bar{V}$ is therefore distributed according to a binomial distribution $P(K=k)=$ $B(k ; \bar{N}, p)$. In a second vector $V$ of length $N$, elements receive 0 or 1 according to a parent element chosen at random in vector $\bar{V}$. This method produces $N$ independent spike trains with Poisson statistics. The correlations emerged due to the fact that $\bar{N}<N$. At each time step, small clusters involving only subsets of the $N$ presynaptic neurons fire synchronously. The probability of receiving a value of 1 is then $\tilde{p}=K / \bar{N}$. The total number of elements 
$Q$ of $V$ receiving a value of 1 is then given by $P(Q=q \mid K)=$ $B(q ; N, \tilde{p})$. The average distribution of variable $Q$ is then:

$P(Q=q)=\sum_{k=0}^{\bar{N}} P(Q=q \mid K) P(K=k)$

Some algebra yields:

$P(Q=q)=C_{q}^{N} \bar{N}^{-N} \sum_{k=0}^{\bar{N}} C_{k}^{\bar{N}} p^{k}(1-p)^{\bar{N}-k} k^{q}(\bar{N}-k)^{N-q}$

In the following, we will need to know the first two moments $E[Q]$ and $\operatorname{Var}[Q]$ of this distribution. Using the definition of $\tilde{p}$ (see above), we find that $E[\tilde{p}]=p$ and thus:

$E[Q]=N p$

To calculate the variance of $Q$, we use the fact that:

$E\left[Q^{2} \mid K\right]=\operatorname{Var}[Q \mid K]+E[Q \mid K]^{2}=N \tilde{p}(1-\tilde{p})+N^{2} \tilde{p}^{2}$

Using the fact that $E[\tilde{p}]=p$ and that $E\left[\tilde{p}^{2}\right]=$ $\bar{N}^{-2} \operatorname{Var}[K]+p^{2}$, we find that:

$\operatorname{Var}[Q]=N p(1-p)(1+N / \bar{N})$

While variables $N$ and $p$ have direct biological interpretations ( $N$ is the size of the presynaptic population and $p$ is related to the discharge frequency in that population), $\bar{N}$ is a rather abstract quantity which is linked to the correlations in the activity of the presynaptic population. For practical use, it would be useful to compute the correlation coefficient between two spike trains in terms of these variables. The correlation coefficient between two sequences of numbers $m_{i}$ and $n_{i}$ is defined by:

$c=\frac{\sum_{i} m_{i} n_{i}}{\sqrt{\sum_{i} m_{i}^{2} \sum_{j} n_{j}^{2}}}$

If we choose at random two elements from the vector $V$, the probability that both fire together is given by the hypergeometric distribution $H(N, q, 2)$ so that:

$P(Y=2 \mid Q)=\frac{C_{2}^{q} C_{0}^{N-q}}{C_{2}^{N}}=\frac{q(q-1)}{N(N-1)}$

On the other hand, if we choose only one element from the vector $V$, the probability that it fires is given by $H(N, q, 1)$ :

$P(Z=1 \mid Q)=q / N$

Finally, the average correlation coefficient between two randomly chosen spike trains of the presynaptic population is given by:

$c=\frac{\sum_{q=0}^{N} P(Y=2 \mid Q) P(Q=q)}{\sum_{q^{\prime}=0}^{N} P(Z=1 \mid Q) P\left(Q=q^{\prime}\right)}$

Using Eqs. (A.7) and (A.8) in Eq. (A.9), we find:

$c=\frac{1}{N-1} \frac{E\left[Q^{2}\right]-E[Q]}{E[Q]}$
Note that the latter result is general when considering homogeneous Poisson spike trains with the same rate $p$ and does not depend on the specific way spike trains are generated. In our case, some algebra yields:

$c=p+\frac{N(1-p)}{\bar{N}(N-1)}$

and thus:

$\bar{N}=\frac{N(1-p)}{(N-1)(c-p)}$

\section{References}

[1] J. Bower, D. Beeman, The Book of Genesis, Springer, New York, 1995.

[2] D. Brillinger, Maximum likelihood analysis of spike trains of interacting nerve cells, Biol. Cyber. 59 (1988) 189-200.

[3] D. Brillinger, J. Segundo, Empirical examination of the threshold model of neuronal firing, Biol. Cyber. 35 (1979) 213-220.

[4] N. Brunel, Dynamics of sparsely connected networks of excitatory and inhibitory spiking neurons, J. Comput. Neurosci. 8 (2000) 183208.

[5] A. Burkitt, H. Meffin, D. Grayden, Study of neuronal gain in a conductance-based leaky integrate-and-fire neuron model with balanced excitatory and inhibitory synaptic input, Biol. Cyber. 89 (2003) 119-125.

[6] A. Destexhe, D. Paré, Impact of network activity on the integrative properties of neocortical pyramidal neurons in vivo, J. Neurophysiol. 81 (1999) 1531-1547.

[7] A. Destexhe, M. Rudolph, D. Paré, The high-conductance state of neocortical neurons in vivo, Nat. Rev. Neurosci. 4 (2003) 739-751.

[8] A. Erisir, D. Lau, B. Rudy, C. Leonard, Specific $\mathrm{K}^{+}$channels are required to sustain high frequency firing in fast-spiking neocortical interneurons, J. Neurophysiol. 82 (1999) 2476-2489.

[9] R. FitzHugh, Impulses and physiological states in models of nerve membrane, Biophys. J. 1 (1961) 445-466.

[10] D. Fricker, R. Miles, EPSP amplification and the precision of spike timing in hippocampal neurons, Neuron 28 (2000) 559-569.

[11] W. Gerstner, Population dynamics of spiking neurons: fast transients, asynchronous states and locking, Neural Comput. 12 (2000) 43-89.

[12] W. Gerstner, W. Kistler, Spiking Neurons Models: Single Neurons, Populations, Plasticity, Cambridge University Press, Cambridge, 2002.

[13] A. Hodgkin, A. Huxley, A quantitative description of ion currents and its applications to conduction and excitation in nerve membranes, J. Physiol. (Lond.) 117 (1952) 500-544.

[14] E. Izhikevich, Simple model of spiking neurons, IEEE Trans. Neural Networks 14 (2003) 1569-1572.

[15] R. Jolivet, T. Lewis, W. Gerstner, Generalized integrate-and-fire models of neuronal activity approximate spike trains of a detailed model to a high degree of accuracy, J. Neurophysiol. 92 (2004) 959976.

[16] J. Keat, P. Reinagel, R. Reid, M. Meister, Predicting every spike: a model for the responses of visual neurons, Neuron 30 (2001) 803-817.

[17] W. Kistler, W. Gerstner, L. van Hemmen, Reduction of HodgkinHuxley equations to a single-variable threshold model, Neural Comput. 9 (1997) 1015-1045.

[18] A. Kuhn, A Aertsen, S. Rotter, Higher-order statistics of input ensembles and the response of simple model neurons, Neural Comput. 15 (2003) 67-101.

[19] L. Lapicque, Recherches quantitatives sur l'excitation électrique des nerfs traitée comme une polarisation, J. Physiol. Pathol. Gen. 9 (1907) 620-635.

[20] Y. Lee, M. Schetzen, Measurement of the wiener kernels of a nonlinear system by cross-correlation, Int. J. Control 2 (1965) 237-254. 
[21] W. Maass, C. Bishop, Pulsed Neural Networks, MIT Press, 1998.

[22] Z. Mainen, T. Sejnowski, Reliability of spike timing in neocortical neurons, Science 268 (1995) 1503-1506.

[23] Z. Mainen, T. Sejnowski, Influence of dendritic structure on firing pattern in model neocortical neurons, Nature 382 (1996) 363-366.

[24] C. Morris, H. Lecar, Voltage oscillations in the barnacle giant muscle fiber, Biophys. J. 35 (1981) 193-213.

[25] J. Nagumo, S. Arimoto, S. Yoshizawa, An active pulse transmission line simulating nerve axon, Proc. IRE 50 (1962) 2061-2070.

[26] J. Nelder, R. Mead, A simplex method for function minimization, Comput. J. 7 (1965) 308-313.

[27] L. Paninski, J. Pillow, E. Simoncelli, Maximum likelihood estimation of a stochastic integrate-and-fire neural encoding model, Neural Comput. 16 (2004) 2533-2561.

[28] A. Rauch, G. La Camera, H. Lüscher, W. Senn, S. Fusi, Neocortical pyramidal cells respond as integrate-and-fire neurons to in-vivo-like input currents, J. Neurophysiol. 90 (2003) 1598-1612.
[29] M. Richardson, The effects of synaptic conductance on the voltage distribution and firing rate of spiking neurons, Phys. Rev. E 69 (2004) 044405 .

[30] H. Robinson, N. Kawai, Injection of digitally synthesized synaptic conductance transients to measure the integrative properties of neurons, J. Neurosci. Methods 49 (1993) 157-165.

[31] M. Rudolph, A. Destexhe, Characterization of subthreshold voltage fluctuations in neuronal membranes, Neural Comput. 15 (2003) $2577-$ 2618.

[32] R. Stein, The information capacity of nerve cells using a frequency code, Biophys. J. 7 (1967) 797-826.

[33] R. Traub, R. Miles, G. Buzsáki, Computer simulation of carbacholdriven rhythmic population oscillations in the CA3 region of the in vitro rat hippocampus, J. Physiol. 451 (1992) 653-672.

[34] N. Wiener, Nonlinear Problems in Random Theory, MIT Press, Cambridge, 1958. 